\title{
Morfoanatomia de la cubierta seminal en siete especies de Passiflora L., subgénero Passiflora (Passifloraceae)
}

\author{
Silvia Peréz-Cortéz ${ }^{1,4}$, Marcia Escala ${ }^{2}$ y Stephen Tillett ${ }^{3}$
}

Recibido: 09.03.2006; aceptado: 26.02.2009

ASBTRACT - (Seed coat morphoanatomy in seven species of Passiflora L., subgenus Passiflora (Passifloraceae)). The morphology and anatomy of the seed coat of seven species of the genus Passiflora L. were studied in order to evaluate the taxonomic value of seed characters in this genus. Three morphological types of seed coat surfaces were found and seven anatomical patterns are species specific, so that, seed coat attributes can be used to distinguish species.

Key words: anatomy, morphology, seed

RESUMEN - (Morfoanatomia de la cubierta seminal en siete especies de Passiflora L., subgénero Passiflora (Passifloraceae)). Se caracterizó morfológica y anatómicamente la cubierta seminal de siete especies del género Passiflora L., con la finalidad de determinar la utilidad taxonómica de los atributos de la semilla en este género. Se encontraron tres tipos morfológicos en la superficie de la cubierta seminal y siete patrones anatómicos diferentes para cada especie. Se concluye que los atributos de la cubierta seminal permiten distinguir especies.

Palabras clave: anatomía, morfología, semilla

\section{Introducción}

Los atributos de la semilla, en muchos casos, son de importancia taxonómica por presentarse con bastante constancia en algunas familias y algunos géneros (Netolitzky 1926, Corner 1976, Lindorf et al. 1985). En la literatura consultada los trabajos de Killip (1938), Raju (1955), Tillett (1988), MacDougal (1994), Perez-Cortéz et al. 1995, 2002, 2005) sobre las características anatómicas y morfológicas de la semilla en especies del género Passiflora señalan que las semillas presentan gran variación en las esculturaciones de la superficie y anatomía de la cubierta seminal y que estas podrían ser de utilidad como herramientas para interpretaciones taxonómicas. El género Passiflora L. está constituido por aproximadamente 500 especies, es el más importante de la familia Passifloraceae (14 géneros, 600 especies) en cuanto al número de especies que agrupa. Killip (1938) divide al género en 22 subgéneros y éstos en secciones y series. La taxonomía del género Passiflora es complicada a causa de las características de la estructura floral y de la variabilidad morfológica de las hojas tanto a nivel de género como de especie e individuo (Tillett 1988, Deginani 2001). Existe un amplio espectro de posibilidades a explorar, para evaluar el valor taxonómico de los atributos de la semilla en el género Passiflora. Esta investigación tiene como finalidad caracterizar la morfoanatomía de la cubierta seminal y evidenciar su utilidad en la taxonomía del género.

\section{Materiales y métodos}

El material utilizado proviene de muestras depositadas en el Herbario Ovalles (MYF) de la Facultad de Farmacia de la Universidad Central de Venezuela, el cual fue identificado por el Dr. Stephen Tillett. Se trabajó con semillas maduras de siete especies (tabla 1).El análisis morfológico de la cubierta seminal se realizó semillas libres de arilo, en 30 muestras por especie con un microscopio estereoscópico (Nikon SMZ-10), consistió en determinar el tipo de esculturación presente.

1. Universidad Central de Venezuela, Fundación Instituto Botánico de Venezuela, Jardín Botánico de Caracas, Av. Salvador Allende. Apartado 2156, Caracas 1010-A, Venezuela

2. Universidad Central de Venezuela, Instituto de Biología Experimental, Centro de Botánica Tropical, Apartado 20513, Caracas, Venezuela

3. Universidad Central de Venezuela, Facultad de Farmacia, Herbario Dr. Víctor M. Ovalles, Apartado 40109, Caracas, Venezuela

4. Autor para la correspondencia: perezsi@gmail.com 
Tabla 1. Especies estudiadas del género Passiflora

Table 1. Studied specie from Passiflora genus

\begin{tabular}{lll}
\hline Especie & Número comprobante & Lugar de recolección \\
\hline P. foetida var. hirsuta Mast. & Tillett, S,.676-401 & Péru \\
P. gritensis Karst. & Molinari s/n & Venezuela-Mérida \\
P. incarnata L. & K.Brown 232 & Alabama \\
P. pallens Peopp. ex Mast. & Tillett, S. 681-2 & EEUU-Florida \\
P. sidaefolia M. Roem. & Tillett, S. 702-25 & Braszil \\
P. spectabilis Killip & Tillett, S.675-385 & Perú \\
P. subpeltata Ortega & Tillett, S. 703-33 & Venezuela-Lara \\
\hline
\end{tabular}

Para el estudio anatómico de la cubierta seminal en secciones transversales se consideraron los siguientes atributos: grosor de la cubierta seminal, número de capas, tipo de capa, tipo de alargamiento radial de las células de cada capa (Rodríguez 1976, Lindorf et al. 1985, Buth et al. 1987, Haridasan \& Mukherjee 1988, Kaur \& Pal 1989, Corral et al. 1989, Small et al. 1990, Escala 1994), las mediciones se realizaron en 25 campos por especie con un microscopio óptico calibrado (Nikon Opthifot 2). El material vegetal fue ablandado en una mezcla de etanol $70 \%$ y glicerina $40 \%$ en relación $1: 1$ por un período de 15 días; luego se hidrató en agua destilada por 24 horas y fue conservado en etanol $70 \%$ por 48 horas (Jha \& Pandey 1989, C. Sánchez, datos no publicados). Posteriormente se realizó la deshidratación gradual en una serie ascendente de alcohol compuesta por alcohol butílico terciario (ABT), glicerina, etanol y agua destilada, colocando la muestra en cada concentración de alcohol por un día. El material deshidratado se infiltró en una mezcla de ABT y parafina en una estufa entre 58 y $60{ }^{\circ} \mathrm{C}$ durante 30 días. Se realizó la inclusión del material en parafina (Johansen 1940). Se realizaron cortes en un micrótomo de rotación a 24 micrómetros. Luego se realizó una coloración diferencial con safraninafast green (Roth 1964). En muchos casos se preparó la lámina sin coloración porque el material se dañaba durante el proceso. Se utilizó entellan como medio de montaje. Se microfotografió de la superficie seminal y de la sección transversal de la cubierta seminal de cada una de las especies estudiadas, empleando una cámara Nikon FX-35DX incorporada al microscopio.

La distribución de las especies está basada en la consulta de la base TROPICOS.

\section{Resultados}

Passiflora foetida L., Sp. Pl.: 959. 1753.

Figuras 1-3

Superficie de la cubierta seminal reticulada, de 234 (128-237) $\mu \mathrm{m}$ de grosor, constituida por cuatro capas de células. La primera capa es uniestrata, formada por células de paredes delgada, que se desprenden, eje principal en posición periclinal. Segunda capa con células de pared engrosada con eje principal en posición periclinal. La tercera capa está formada por macroesclereidas con patrón de alargamiento radial levemente sinuoso. Cuarta capa de células obliteradas.

Distribución: Ecuador, Nicaragua, Perú, China, Venezuela (Amazonas, Anzoátegui, Apure, Bolívar, Carabobo, Distrito Federal, Lara, Mérida, Miranda, Monagas, Sucre, Yaracuy, Zulia).

Passiflora gritensis Karst., Linnaea 3: 163. 1859.

Figuras 4-6

Superficie de la cubierta seminal escrobiculada, 202 (198-206) $\mu \mathrm{m}$ de grosor, constituida por cuatro capas de células. La primera capa es uniestrata, formada por células de pared delgada, con contenido celular aparente. En la segunda capa las paredes anticlinales y periclinal interna están engrosadas, confiriendo a la célula forma de "U".La tercera capa está formada por macroesclereidas con patrón de alargamiento radial levemente sinuoso. Cuarta capa de células obliteradas.

Distribución: Venezuela (Mérida y Táchira), Colombia (Norte de Santander)

Passiflora incarnata L., Sp. Pl. 2: 959. 1753.

Figuras 7-9 


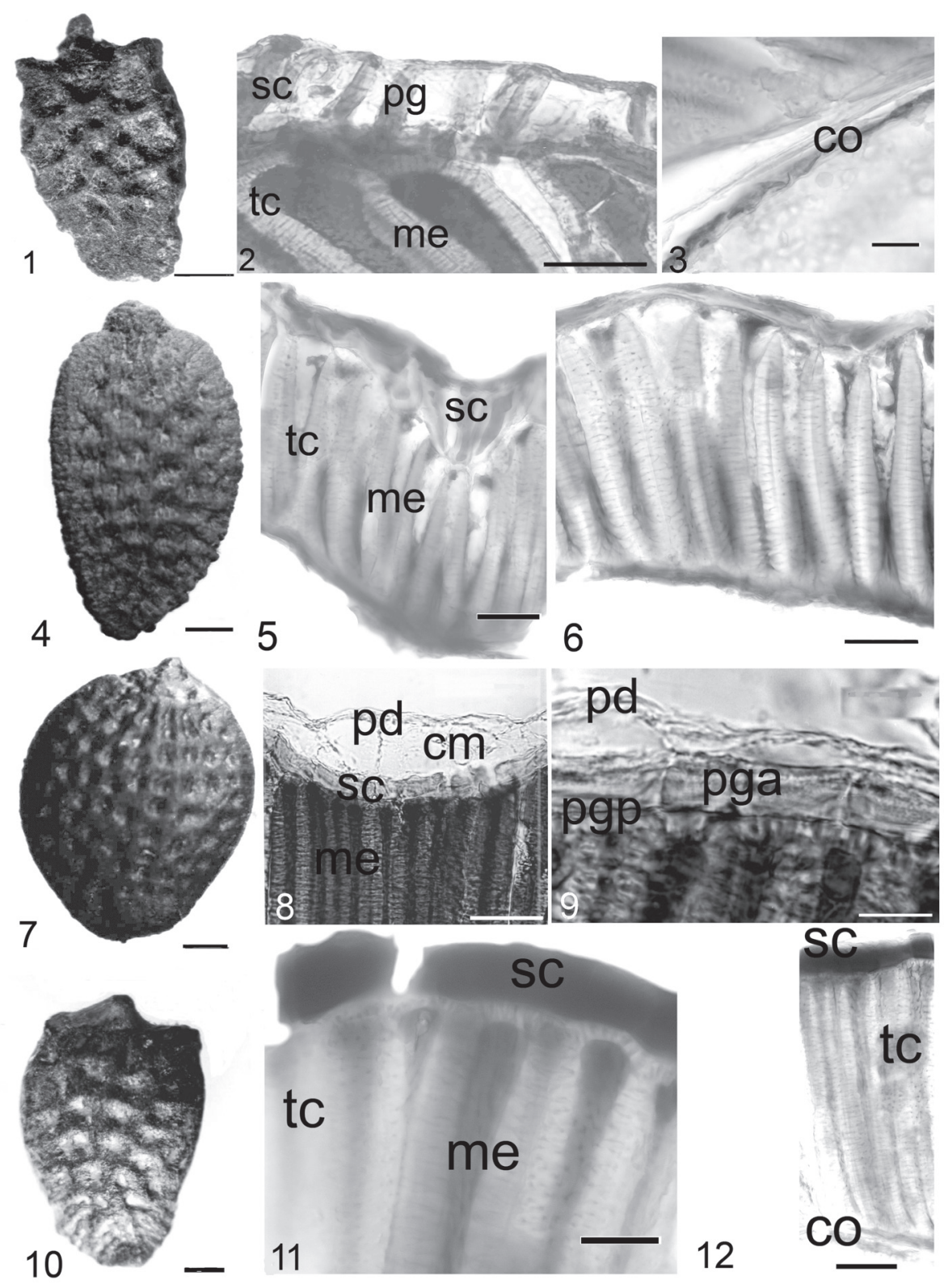

Figuras 1-12. Morfología y anatomía de la cubierta seminal en especies del género Passiflora. 1-3. P.foetida var. hirsuta 1. Semilla completa con superficie reticulada. 2. Células de la segunda capa con células de pared gruesa (pg), capa de macroesclereidas (me) con células de pared gruesa (pg). 3. Cuarta capa de células obliteradas (co). 4-6. P. gritensis. 4. Semilla completa con superficie escrobiculada. 5. Segunda capa (sc), las células de la segunda aumentan de tamaño en el valle, tercera capa (tc) de macroesclereidas (me). 6. Tercera capa (tc) de macroesclereidas (me) con alargamiento radial levemente sinuoso. 7-8. P. incarnata. 7. Semilla completa con superficie retículo-foveada. 7. Células de la primera capa de paredes delgadas (pd), capa multiplicativa (cm), células de la segunda capa (sc), macroesclereidas (me). 9. Detalle de las paredes anticlinales (pga) y periclinal interna (pgp) engrosada en la segunda capa, pared gruesa de las macroesclereidas (pg). 10-12. P. pallens. 10. Semilla completa con superficie retículo-foveada. 11. Células de la segunda capa (sc) con paredes delgadas (pd), paredes gruesas (pg) de las macroesclereidas (me). 12. Segunda, tercera y cuarta capa, la cuarta con células obliteradas (co). Escalas: figuras 1, 4,7,10 =1 cm; figuras 2,8,12=50 $\mu \mathrm{m}$; figuras 5, $6=100 \mu \mathrm{m}$; figuras 3, 9, $11=16,6 \mu \mathrm{m}$.

Figures 1-12. Morphology and anatomy of the seminal coat in species of the genus Passiflora. 1-3. P. foetida var. hirsuta 1. Seed with reticulate surface. 2. Second layer with thick-walled cells (pg), layer of macrosclereids (me) with thick-walled cells (pg). 3. Fourth layer of obliterated cells (co). 4-6. P. gritensis. 4. Seed with escrobiculate surface. 5. Second layer (sc), the cells of second layer increase of long in the valley, third layer (tc) of macrosclereids (me). 6. Third layer (tc) of macrosclereids (me) with slightly sinuose radial extension. 7-9. P. incarnata. 7. Seed with surface reticule-foveate. 8. First layer of thin-walled cells (sc), layer multiplicative $(\mathrm{cm})$, cells of the second layer (sc), macrosclereids (me). 9. Detail of the anticlinal walls (pga) and internal periclinal wall (pgp) thickened in the second layer, thick wall of the macrosclereids (pg). 10-12. P. pallens. 10. Seed with surface reticule-foveate. 11. Cells of the second layer (sc) with thin-walled (pd), thick walls (pg) of the macrosclereids (me). 12. Second, third and fourth layer, the fourth layer with cells obliterated (co). Scales: figures 1, 4, 7, $10=1 \mathrm{~cm}$; figures 2, 8, $12=50 \mu \mathrm{m}$; figures 5, $6=100 \mu \mathrm{m}$; figures 3, 9, $11=16,6 \mu \mathrm{m}$. 


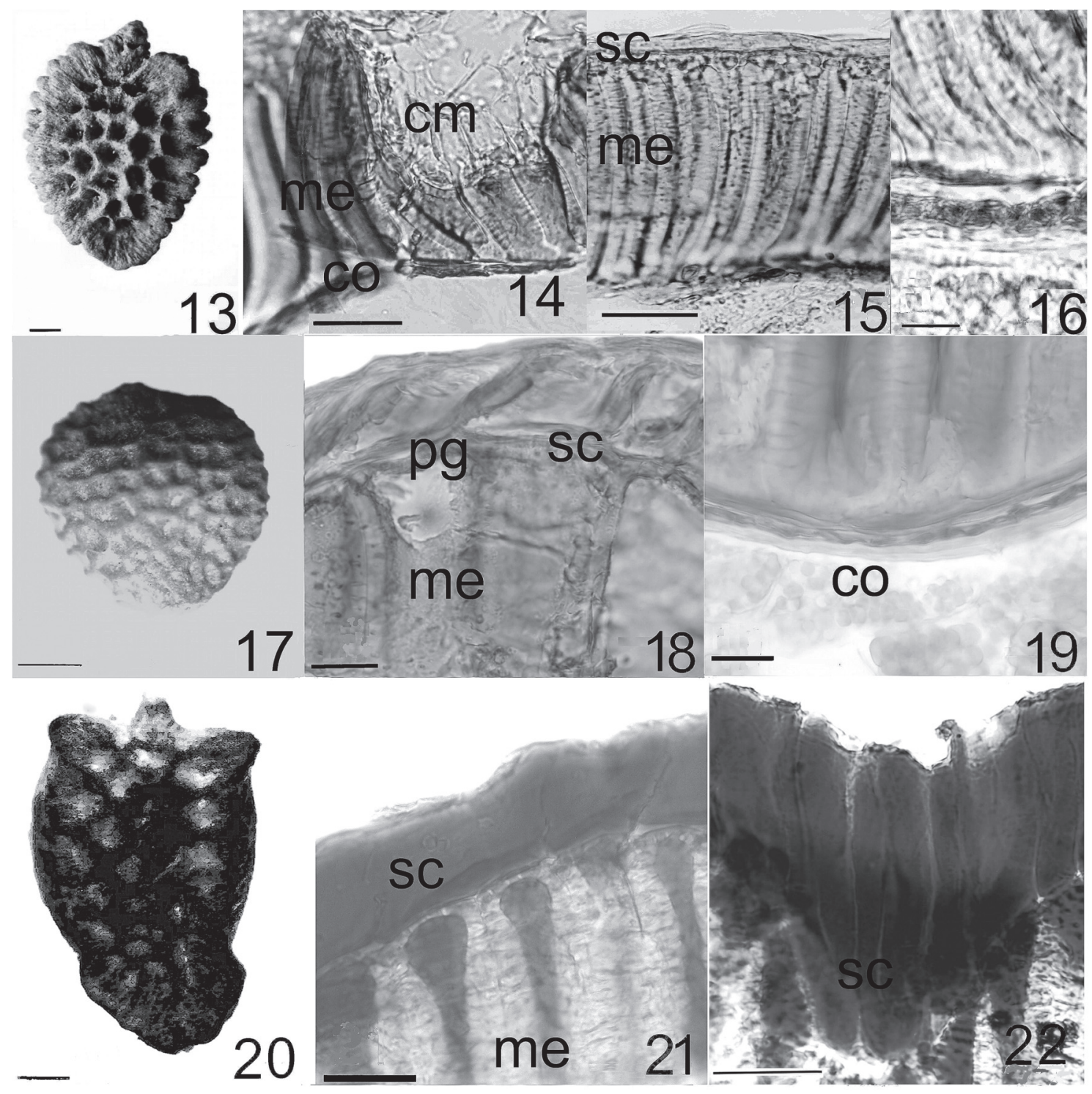

Figuras 13-22. Morfología y anatomía de la cubierta seminal en especies del género Passiflora. 13-16. P. sidiifolia. 13. Semilla completa con superficie retículo-foveada. 14. Primera capa multiplicativa $(\mathrm{cm})$ con células de paredes delgadas (pd), células de la segunda capa con contenido celular aparente (con), macroesclereidas (me). 15. Células de la segunda capa con paredes delgadas (pd), capa de macroesclereidas (me). 16. células obliteradas (co) de la cuarta capa. 17-19. P. spectabilis. 17. Semilla completa con superficie reticulada. 18. Segunda capa (sc), tercera capa de células (tc) de macroesclereidas (me). 19. Células obliteradas (co) de la cuarta capa. 20-22. P. subpeltata. 20. Semilla completa con superficie retículo-foveada. 21. Segunda capa con contenido celular aparente y tercera capa (tc) de macroesclereidas. 22. Aumento de tamaño de las células de segunda capa en la zona de valle. Escalas: figuras $13,17,20=1 \mathrm{~cm}$; figuras $14,15,18,21,22=50 \mu \mathrm{m}$; figuras $16,19=16,6 \mu \mathrm{m}$.

Figures 13-22. Morphology and anatomy of the seminal coat in species of the Passiflora genus. 13-16. P. sidaefolia. 13. Seed with surface reticule-foveate. 14. Multiplicative first layer $(\mathrm{cm})$ with thin-walled cells (pd), cells of the second layer with cellular content (con), macrosclereids (me). 15. Second layer with thin-walled cells (pd), layer of macrosclereids (me). 16. Obliterated cells (co) of the fourth layer. 17-19. P. spectabilis. 17. Seed with reticulate surface. 18. Second layer (sc), third layer of cells (tc) of macrosclereids (me). 19. Obliterated cells (co) of the fourth layer. 20-22. P. subpeltata. 20. Seed with surface reticule-foveate. 21. Second layer with macrosclereids cellular content and third layer (tc) of macrosclereids. 22. Increase of long of the cells of second layer in the valley. Scales: figures 13 , $17,20=1 \mathrm{~cm}$; figures $14,15,18,21,22=50 \mu \mathrm{m}$; figures $16,19=16,6 \mu \mathrm{m}$. 
Superficie de la cubierta seminal finamente retículo-foveada, de 400 (399-408) $\mu \mathrm{m}$ de grosor, constituida por cuatro capas de células. La primera capa es multiplicativa, con células de pared delgada, con eje principal en posición periclinal, en los valles el eje principal células es anticlinal. En la segunda capa las paredes anticlinales y periclinal interna están engrosadas, confiriendo a la célula forma de "U". La tercera capa está formada por macroesclereidas con patrón de alargamiento radial sinuoso. Cuarta capa de células obliteradas.

Distribución: Estados Unidos (Alabama, Arkansas, North Carolina, South Carolina, Florida, Georgia, Illinois, Kentucky, Louisiana, Mississippi, Missouri, Tennessee, Texas, Washington, Virginia).

Passiflora pallens Poepp. ex Mast, Trans. Linn. Soc. London 27(4): 638. 1871.

Figuras 10-12

Superficie de la cubierta seminal retículo-foveada, de 255 (250-256) $\mu \mathrm{m}$ de grosor, constituida por cuatro capas de células. La primera capa es uniestrata, formada por células de paredes delgadas. La segunda capa con células de pared delgada y contenido celular aparente. La tercera capa está formada por macroesclereidas con patrón de alargamiento radial homogéneo. Cuarta capa de células obliteradas.

Distribución: Estados Unidos (Florida), Cuba (La Habana, Pinar del Río, Santa Clara).

Passiflora sidiifolia M. Roem., Fam. Nat. Syn. Monogr.: 173. 1846.

Figuras 13-16

Superficie de la cubierta seminal retículo-foveada, de 175 (172-178) $\mu \mathrm{m}$ de grosor, constituida por cuatro capas de células. La primera capa es multiplicativa, formada por células de pared delgada, estas células aumentan sus dimensiones en las zonas de valle de la cubierta seminal. La segunda capa con células de pared delgada y contenido celular aparente. La tercera capa está formada por macroesclereidas con patrón de alargamiento radial sinuoso. Cuarta capa de células obliteradas.

Distribución: Brasil (Sureste), Perú.

Passiflora spectabilis Killip, J. Wash. Acad. Sci. 20: 379. 1930.

Figuras 17-19

Superficie de la cubierta seminal reticulada, de 512 (504-515) $\mu \mathrm{m}$ de grosor, constituida por cuatro capas de células. La primera capa es multiplicativa, formada por células de pared delgada. En la segunda capa las paredes anticlinales y periclinal interna están engrosadas, confiriendo a la célula forma de "U". La tercera capa está formada por macroesclereidas con patrón de alargamiento radial sinuoso. Cuarta capa de células obliteradas.

Distribución: Perú (Amazonas, Loreto, Pasco), Brasil (Maranhão)

Passiflora subpeltata Ortega, Nov. Pl. Descr. Dec. 6: 78.1798.

Figuras 20-22

Superficie de la cubierta seminal retículo-foveada, de 534 (532-538) $\mu \mathrm{m}$ de grosor, constituida por cuatro capas de células. La primera capa es multiplicativa, formada por células de pared delgada, estas células aumentan sus dimensiones en las zonas de valle de la cubierta seminal. En la segunda capa las células presentan contenido celular aparente, cuyo eje anticlinal aumenta de longitud en la zona de .valle. La tercera capa está formada por macroesclereidas con patrón de alargamiento radial sinuoso. Cuarta capa de células obliteradas.

Distribución: Guatemala, Panamá (Canal de Panamá, Cririqui, Colón), México (Chiapas, Distrito Federal, Hidalgo, Jalisco, Oxacas, Puebla, Queretaro, Veracruz), Venezuela (Bolívar, Falcón, Aragua, Miranda, Zulia), Colombia (Antioquia, Santa Marta, Cundinamarca, Tulima).

\section{Discusión}

En algunos taxa el patrón anatómico de la cubierta seminal es uniforme mientras que en otros es variable pudiendo ser útil para interpretaciones taxonómicas (Netolitzky 1926, Corner 1976, Johri 1984, Werker 1997). La caracterización morfoanatomica de la cubierta seminal en las especies estudiadas del género Passiflora, subgénero Passiflora, series Lobatae (P. gritensis, P. pallens, P. spectabilis, P. subpeltata), Dysosmia (P. foetida), Incarnatae (P. incarnata) e Imbricatae (P. sidiifolia) (Killip 1938), muestra que el patrón anatómico de la cubierta es especie específico y que la ornamentación de la cubierta permite formar grupos. Esto se corresponde con lo indicado por Deginani (2001), Pérez-Cortéz et al. (1995, 2002, 2005), quienes señalan el valor diagnóstico de la semilla en especies de este género.

Killip en 1938 encontró que los caracteres de la cubierta seminal en especies de Passiflora, subgénero Plectostemma, están asociados con el tipo de pecíolo y 
éste es de importancia para la clasificación de especies, así las especies sin glándulas peciolares tienen semillas con superficie seminal transversalmente surcada y en especies con pecíolos glandulíferos la superficie es reticulada o alveolada. Deginani (2001) describió las especies argentinas de Passiflora y encontró que la morfología de la cubierta seminal contribuyó en la identificación de especies.

La agrupación de las especies estudiadas de acuerdo al patrón de alargamiento radial de las macroesclereidas y a la condición multiplicativa o uniestrata de la primera capa no están asociados con las series del subgénero Passiflora propuestas por Killip (1938). La condición multiplicativa de la primera capa podría estar asociada al patrón de alargamiento radial sinuoso, ya que siempre se presenta aunada a éste.

Trabajos sobre la anatomía de la cubierta seminal en especies del género Passiflora señalan que está constituida por una o dos capas externas de células transparentes, una capa media de macroesclereidas y una capa interna de células obliteradas (Raju 1955, Martín \& Barkley 1973, Corner 1976, Pérez-Cortéz et al. 1995, 2005). La cubierta seminal en especies del género Passiflora es formada por dos tegumentos, del tegumento externo se originan la capa más externa de cubierta seminal y la capa multiplicativa; del tegumento interno se origina la capa de macroesclereidas y la capa de células obliteradas (Raju 1955). La capa de macroesclereidas y la capa de células obliteradas están presentes en el patrón anatómico de la cubierta seminal de las especies estudiadas en esta investigación como en las especies reportadas en la literatura, por lo tanto ambas capas podrían ser comunes a todas las especies del género y probablemente características de éste. De acuerdo a Raju (1955) en la descripción anatómica de la cubierta seminal de Adenia venenata (Passifloraceae) la capa de macroesclereidas está ausente.

La morfología foliar, ampliamente empleada en taxonomía, en las especies del género Passiflora tiene alto grado de polimorfismo, probablemente el mayor de todos los géneros de angiospermas (Deginani 2001), esto ha propiciado la descripción de numerosas especies que luego se han reducido a sinonimia. El polimorfismo está ausente en las características de la semilla consideradas para describir las especies estudiadas del género Passiflora.

Las características morfoanatómicas de la cubierta seminal contribuyen ampliamente en la identificación de especies, aún cuando no explican las relaciones infragenéricas establecidas por Killip (1938). En conclusión se puede señalar que el patrón morfoanatómico de la cubierta seminal en el género Passiflora es característico de cada especie.

\section{Agradecimientos}

Agradecemos por el financiamiento de esta investigación al Postgrado en Botánica de la Facultad de Ciencias de la Universidad Central de Venezuela, al FONACIT y al proyecto CDCH \# 03025.96.

\section{Literatura citada}

Buth, G.M., Roshan, A. \& Narayan, A. 1987. Seed and seed coat anatomy of some members of tribe Arabideae (Brassicaceae). Phytomorphology 37: 341-348.

Corner, E.J.H. 1976. The seeds of Dicotyledons. v. 1, v. 2. Cambridge University Press, Cambridge.

Corral, R., Pérez-García, F. \& Pita, J.M. 1989. Seed morphology and histology in four species of Cistus L. (Cistaceae). Phytomorphology 39: 75-80.

Deginani, N.B. 2001. Las especies argentinas del género Passiflora (Passifloraceae). Darwiniana 39: 43-129.

Escala, M. 1994. Estudio morfoanatómico de frutos y semillas de leguminosas de los altos Llanos Centrales de Venezuela (Estación Biológica de los Llanos de la Sociedad Venezolana de Ciencias Naturales). Boletín de la Sociedad de. Ciencias Naturales La Salle 148:260-316.

Haridasan, V.K. \& Mukherjee, P.K. 1988. Seed surface features of some members of the Indian Campanulaceae. Phytomorphology 37: 277-285.

Holst, B.K. 2008. Myrtaceae. In: Nuevo catálogo de la flora vascular de Venezuela (O. Hokche, P. Berry \& O. Huber, eds.). Fundación Instituo Botánico de Venezuela, Caracas, pp. 1-859.

Jha, S.S. \& Pandey, A.K. 1989. Seed coat structure in Melilotus (Fabaceae) Phytomorphology 39: 221-229.

Johansen, D.A. 1940. Plant microtechnique. Mc GrawHill, New York.

Johri, B.M. 1984. Embryology of angiosperms. SpringerVerlag, Berlin.

Kaur, H. \& Pal, A. 1989. Structure, anatomy and spermoderm pattern seeds in some Vicia species (Papilionoideae). Phytomorphology 39: 363-370.

Killip,E.P. 1938. The American species of Passifloraceae. Field Museum Natural History, Botany Series 19: 1-613.

Lindorf, H., de Parisca, L.\& Rodríguez, P. 1985. Botánica: Clasificación, estructura y reproducción. Ediciones de la Biblioteca UCV, Caracas.

MacDougal, J.M. 1994. Revision of Passiflora Subgenus Decaloba, secction Pseudodysosmia (Passifloraceae). Systematic Botany Monographic 41: 1-46.

Martin, A.C. \& Barkley, W. 1973. Seed identification manual. University of California Press, Barkley.

Netolitzky, F. 1926. Anatomie der Angiospermen Samen. Handbuch der Pflanzenanatomie. Gebrüder Borntraeger, Berlin. 
Tropicos. 2005. Missouri Botanical Garden. http://mobot. org $/ \mathrm{w} 3 \mathrm{t} / \mathrm{search} / \mathrm{vast.thml}$. (acceso en 13.2. 2005).

Pérez-Cortéz, S., Escala, M., Tillett, S. \& Sánchez, C. 1995. Estudio morfoanatómico de la cubierta seminal de Passiflora quadrangularis L. (Passifloraceae). Anales Botánica Agrícola 2: 25-29.

Pérez-Cortéz, S., Tillett, S. \& Escala, M. 2002. Estudio morfológico de la semilla de 51 especies del género Passiflora L. Acta Botanica Venezuelica 25: 67-96.

Pérez-Cortéz,S., Escala, M.\& Tillett,S. 2005. Anatomía de la cubierta seminal en ocho especies del género Passiflora L., subgénero Passiflora. Acta Botanica Venezuelica 28: 337-348.

Raju, M.V.S. 1955. Embryology of the Passifloraceae. I. Gametogenesis and seed development of Passiflora calcarata Mast. Journal of Indian Botany 35: 126-138.
Rodríguez,P. 1976. Estudio sobre los frutos carnosos y sus semillas en las Rubiaceae de Venezuela. Acta Botanica Venezuelica 11: 283-383.

Roth, I. 1964. Microtecnia vegetal. Ediciones de la biblioteca UCV, Caracas.

Tillett, S.S. 1988. Passionis passifloris II.Terminología. Ernstia 48: 1-40.

Small, E., Brookes, B. \& Lassen, P. 1990. Circumscription of genus Medicago (Leguminosae) by seeds characters. Canadian Journal of Botany 68: 613-629.

Werker, E. 1997. Seed anatomy. Encyclopedia of plant anatomy. Tomo X. 3. Gebrüder Borntraeger, Berlin. 